\title{
Epileptic Seizure Prediction and Source Localization using Entropy Analysis of Scalp EEG
}

\author{
Mohammad Ali Reza and A S M Shamsul Arefin* \\ Department of Biomedical Physics and Technology, University of Dhaka, Dhaka - 1000, Bangladesh \\ * Corresponding Author email: arefin.bmpt@du.ac.bd
}

\begin{abstract}
Epilepsy is one of most common neurological disorders that affects people of all ages and can cause unpredictable seizures which may even cause death. The prediction of epileptic activities thus can have a great impact in avoiding fatal injuries through early preparation with medicines and also in improving the efficacy of medicines. A technique for early prediction of epileptic seizure from EEG signal is proposed in this paper. The pre-ictal period of epileptic seizure clearly depicts a start of seizure and comparing the changes in entropy of EEG signals in different brain regions during the pre-seizure period, the proposed technique could successfully predict the seizure using entropy analysis. Moreover, the region of the epileptic activities was also localized by dividing the total brain into four topographic regions and by calculating the entropy from this four zones separately. Thus, this proposed technique has the potential to help the clinical neurologists to investigate seizure detection and treat the patient in a better way with less supervision and better accuracy.
\end{abstract}

Keywords: EEG, Epileptic Seizure, Pre-ictal period, Shannon wavelet entropy.

\section{INTRODUCTION}

According to the World Health Organization (WHO), approximately 50 million people worldwide are suffering from epilepsy which constitutes $1 \%$ of the global diseases. $80 \%$ of the epileptic cases are from developing world, and in some areas $80-90 \%$ of the patients do not receive any treatment (WHO, 2005). Epilepsy is a special type of anomaly in brain function, predominantly by unpredictable and periodic interruptions of normal electrical activities of brain, called epileptic seizures. The International League Against Epilepsy (ILAE) defines epilepsy as:

- "At least two unprovoked (or reflex) epileptic seizures occurring more than 24 hours apart.

- One unprovoked (or reflex) epileptic seizure, and a probability of further epileptic seizures similar to the general recurrence risk (at least 60 percent) after two unprovoked seizures, occurring over the next 10 years diagnosis of an epilepsy syndrome." 
The electrical changes in brain region is depicted by the signal anomaly. The behavior of electroencephalogram changes rapidly in seizure period. And this random irregularity have abnormal impact on the normal brain function (Teplan, 2002). This abnormal excessive or synchronous neuronal activity in the brain can cause lapse of attention, whole body convulsion, sustaining physical injuries and even death. Fortunately, the seizure can be anticipated from this anomaly of the pre seizure period signal. The features extracted from the EEG signal are the factors behind the anticipation. Epileptic Seizure can be predicted by analyzing the EEG pre-ictal period of epilepsy which normally starts 30 second preceding the seizure period (Chaudhary, et al., December, 2012). The early prediction has two valuable impacts, one is to avoid fatal injuries with the early preparation with medicines, and the other is to increase the effect of medicines (Ramgopal, et al., 2014). However, Twenty percent of epilepsies are drug resistant (Dollahpour \& Jalilifar, 2014). Hence, these patients can only be treated with surgery rather than drug or medication alone. And thus in the context of surgery, localization of brain region responsible for epileptic seizure is another necessity.

Generally by identifying the abrupt nature of the signal, the seizure can be predicted. But this process requires a continuous monitoring by another person and has a higher probability of error. Hence, to make the process of onset prediction completely computerized, multiple automation techniques based on signal processing have been the topic of research. There are various types of linear as well as nonlinear methods used for early prediction of epileptic seizure. The linear methods include phase synchronization whereas the nonlinear techniques are Correlation integral, Dimension, Lyapunov exponent, entropy etc. In addition, measuring the spike rate of intracranial EEG signal, the seizure can be predicted (Shufang, et al., 2013). Furthermore, the beta band power analysis of EEG signal has been performed in some previous research works for predicting seizure (Ahmad, et al., 2013). In biomedical field, entropy measurement to assess the complexity of signal is getting popular recently, as the natural and physiologically meaningful features can be obtained by capturing the feature of how chaotic a signal gets with time ( $\mathrm{Li}$, et al., 2018).

In this context, this work aimed to develop a technique to predict the epileptic seizure as well as to localize the source of epileptic activity. The pre-recorded multichannel EEG data were separated according to four different zones of brain and pre-ictal and ictal EEG signals were decomposed with wavelet transformation. Furthermore, the entropy of wavelet coefficients were measured and compared among different channels for four different regions to predict epileptic seizure and localize the source of epileptic activities in the brain. 


\section{METHODOLOGY}

\section{EEG Dataset}

The EEG dataset available on CHB-MIT Scalp EEG database provided by Physionet was used in this work (Goldberger, et al., 2000). There were 23 channels of EEG signals for each epileptic patients. 23 electrodes were positioned according to the international 10-20 system. Recordings, grouped into 24 cases, were collected from 23 subjects, there were 5 males and 17 female subjects while 1 is unknown. The signals were sampled at a rate of $256 \mathrm{~Hz}$ and with 16-bit resolution. Most files contained 23 EEG channels (24 or 26 in a few cases). Each channel represents the bipolar EEG measurement taken from the reading of a pair of electrodes. The records include total 198 seizure events from 916 hours of continuous measurement of EEG. Line noise was removed from the signals already (Goldberger, et al., 2000).

\section{Computational Framework}

The EEG signal of pre-ictal period and non-ictal period were separated and decomposed by wavelet transformation up to 5th level of details. As the EEG signals had been sampled with $256 \mathrm{~Hz}$, and the noise had already been removed, decomposing the signal into five level of details can subdivide the frequency bands successfully. Then the twenty three channel data were grouped into 4 different groups according to the four different zones of brain as shown in Fig. 1. Sequentially the entropies were calculated for ictal and pre-ictal periods and they were compared to predict and localize the seizure activities. Data analysis and interpretation were done via MATLAB and Microsoft Excel. A simple block diagram as in Fig. 2 illustrates the methodological framework.

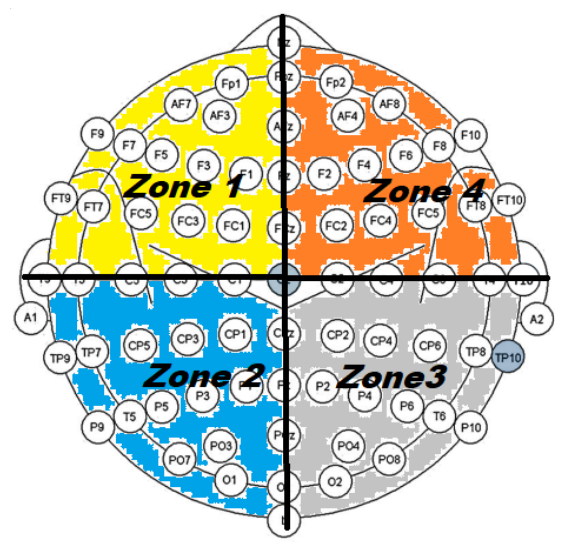

Figure 1. A 10-20 EEG system divided into four zones. 


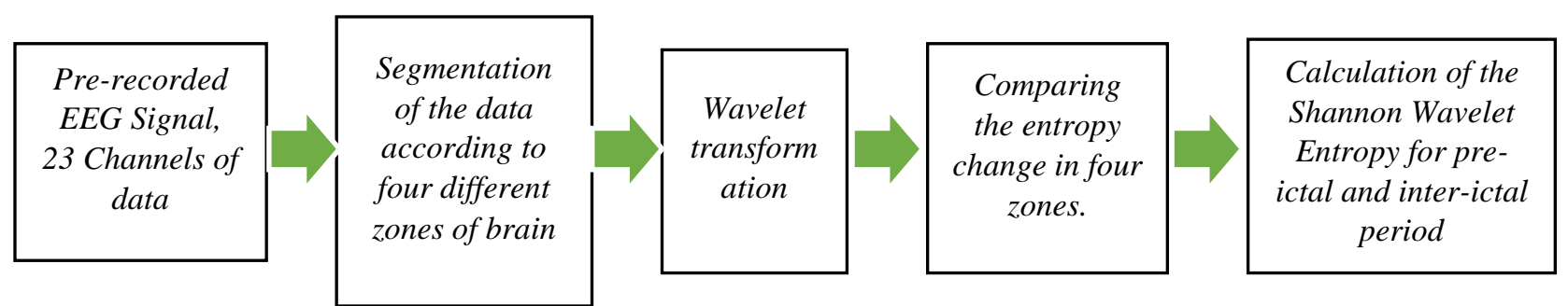

Figure 2. Computational framework for the prediction of epilepsy and localization of epileptic source.

\section{Entropy Analysis Method}

Shannon wavelet entropy was measured for the pre-ictal and non-ictal signal. The signal was sampled into $\mathrm{N}$ number of values with a sampling time $\Delta t=\frac{1}{256} \mathrm{~s}$. 'Daubechies 4' was used as the mother wavelet and the signal is decomposed through all the resolution levels from 1 to $j=1$ to $j_{\max }=5$.

The wavelet expansion of the signal is,

$$
f(x) \cong \sum_{\mathrm{j}=1}^{j_{\max }} \sum_{k} C_{j, k} \psi_{j, k}(x)
$$

where, $C_{j, k}$ is the wavelet coefficient and $\mathrm{F}=\left\{\psi_{j, k}(x)\right\}$ is the orthonormal basis of wavelet transformation.

The Shannon Wavelet Entropy (Shannon, 1948) is a very useful tool to measure and analyze the information contained in any probability distribution.

$$
\text { Shannon Wavelet Entropy, } S\left[\mathrm{P}^{w}\right]=-\sum_{\mathrm{j}=1}^{j_{\max }} \rho_{j} \log _{2}\left(\rho_{j}\right)
$$

where,

$$
\text { Relative Wavelet Energy, } \rho_{j}=\frac{\text { Energy at jth resolution level, } E_{j}}{\text { Total energy, } E_{\text {tot }}}
$$

$E_{j}$ and $E_{t o t}$ are defined as,

$$
\begin{gathered}
E_{j}=\sum_{j}\left|C_{j, k}\right|^{2} \\
E_{\text {tot }}=\sum_{j=1}^{j_{\max }} E_{j}=\sum_{\mathrm{j}=1}^{j_{\max }} \sum_{j}\left|C_{j, k}\right|^{2}
\end{gathered}
$$




\section{RESULTS AND DISCUSSIONS}

The EEG signals were decomposed with wavelet transformation with five level of decomposition. Fig. 3 shows the non-ictal, pre-ictal and ictal period signal of 23 channels of a patient. As the seizure period approaches, the signal tends to show increment in amplitudes and the introduction of spikes arises. This period is called pre-ictal period and is marked in brown. The non-ictal period, marked in gray, has the difference with the pre-ictal period in terms of amplitudes. The ictal (seizure) period, marked in white has the continuous spikes and shows rapid increase of neural electrical activities.

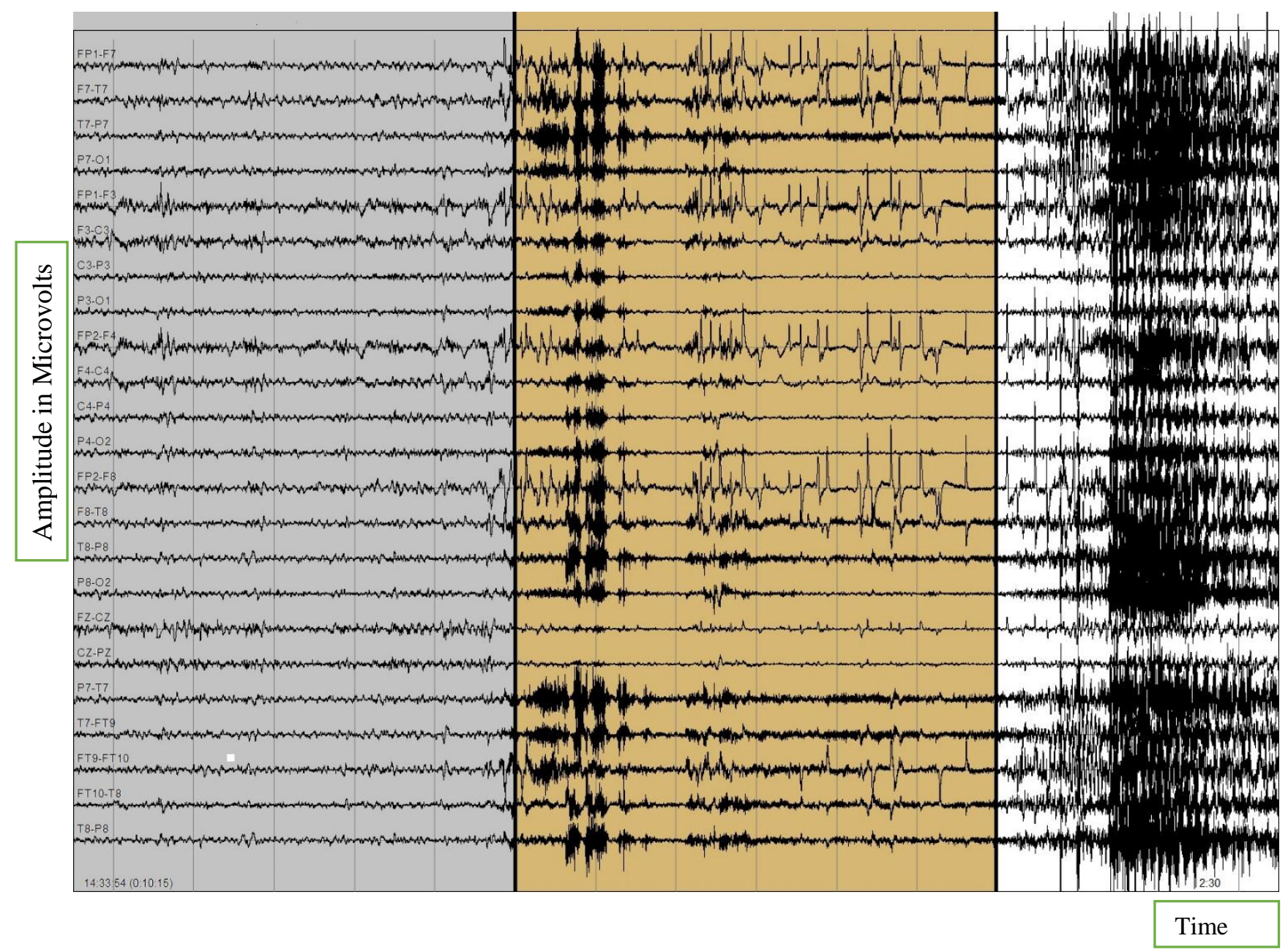

Figure 3. 23 Channel EEG of an Epileptic Patient showing non-ictal (grey background), pre-ictal (brown background) \& ictal (white background) period. 
The abrupt change in EEG signal patterns for a particular channel can be recognized visually as depicted in Fig. 4. Here, FP1-F7 channel of a patient displays non-ictal (blue), pre-ictal (red) and ictal (black) EEG signal distinguishably. The epileptic seizure was transient and there were twenty three channels of data to be analyzed where any of them could have been contaminated with noise or abnormality. Hence, to perform the onset prediction properly, the multichannel data were separated according to four different zones of brain. If we do not subdivide the channels according to the topographic regions and calculate the overall entropy for all the channels, the difference would not be significantly distinguishable as some distant channels, away from the region of seizure location, might not show any difference between pre-ictal and non-ictal period. Thus, this would enhance the chance of identifying the difference between entropies of pre-ictal and non-ictal period. Afterwards entropies were calculated separately for the different channels and sequentially the arithmetic mean of each zone was calculated to rule out the chance of measurement error.

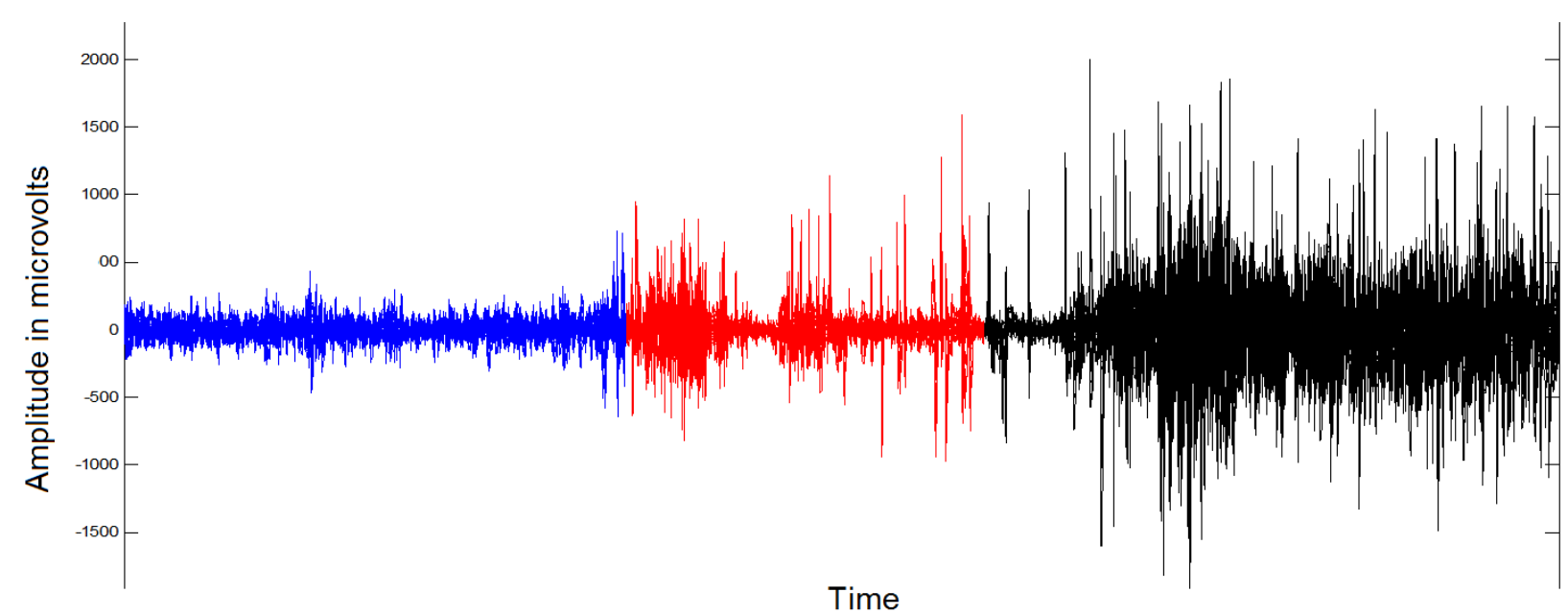

Figure 4. EEG signal of a patient from FP1-F7 channel: non-ictal (blue), pre-ictal (red) and ictal (black).

The entropy of wavelet coefficients were measured for approximately every 100s duration for the different channels. Table 1 portrays a comparative analysis of the data of non-ictal and pre-ictal period of ten epileptic patients. The change of entropy was significantly higher in a specific zone (highlighted cells in Table 1), indicating the part of the brain responsible for the epileptic seizure. The total procedure was an onset prediction. So the entropies were calculated for approximately every 100s duration. 
Table 1. Difference between the entropies of ictal and non-ictal period of ten epileptic patients.

\begin{tabular}{|l|l|l|l|l|}
\hline & \multicolumn{4}{|l}{ Difference between the entropies of pre-ictal and non-ictal period } \\
\hline & Zone 1 & Zone 2 & Zone 3 & Zone 4 \\
\hline Patient 1 & 0.0043 & 0.0110 & 0.0040 & 0.0051 \\
\hline Patient 2 & 0.0015 & 0.0045 & 0.0127 & 0.0054 \\
\hline Patient 3 & 0.0045 & 0.0154 & 0.0075 & -0.0040 \\
\hline Patient 4 & 0.0015 & 0.0112 & 0.0025 & 0.0067 \\
\hline Patient 5 & 0.0054 & 0.0065 & 0.0108 & 0.0037 \\
\hline Patient 6 & 0.0025 & 0.0001 & 0.0054 & 0.0150 \\
\hline Patient 7 & 0.0069 & 0.0101 & 0.0000 & 0.0054 \\
\hline Patient 8 & 0.0032 & 0.0120 & 0.0025 & 0.0075 \\
\hline Patient 9 & 0.0105 & 0.0012 & 0.0059 & 0.0054 \\
\hline Patient 10 & 0.0048 & 0.0015 & 0.0007 & 0.0097 \\
\hline
\end{tabular}

To illustrate the analysis, for instance, the difference between two 100s duration signal entropies of nonictal period of patient 1 was measured and found to be nearly zero for every zone. Whereas, measuring the pre-ictal period entropy and comparing with the non-ictal signal entropy, the difference was substantial and Zone 2 had the abrupt change in entropy. This sudden change lead us to predict the seizure and to localize the region of epileptic activities. The data from Table 1 was plotted in Fig. 5.

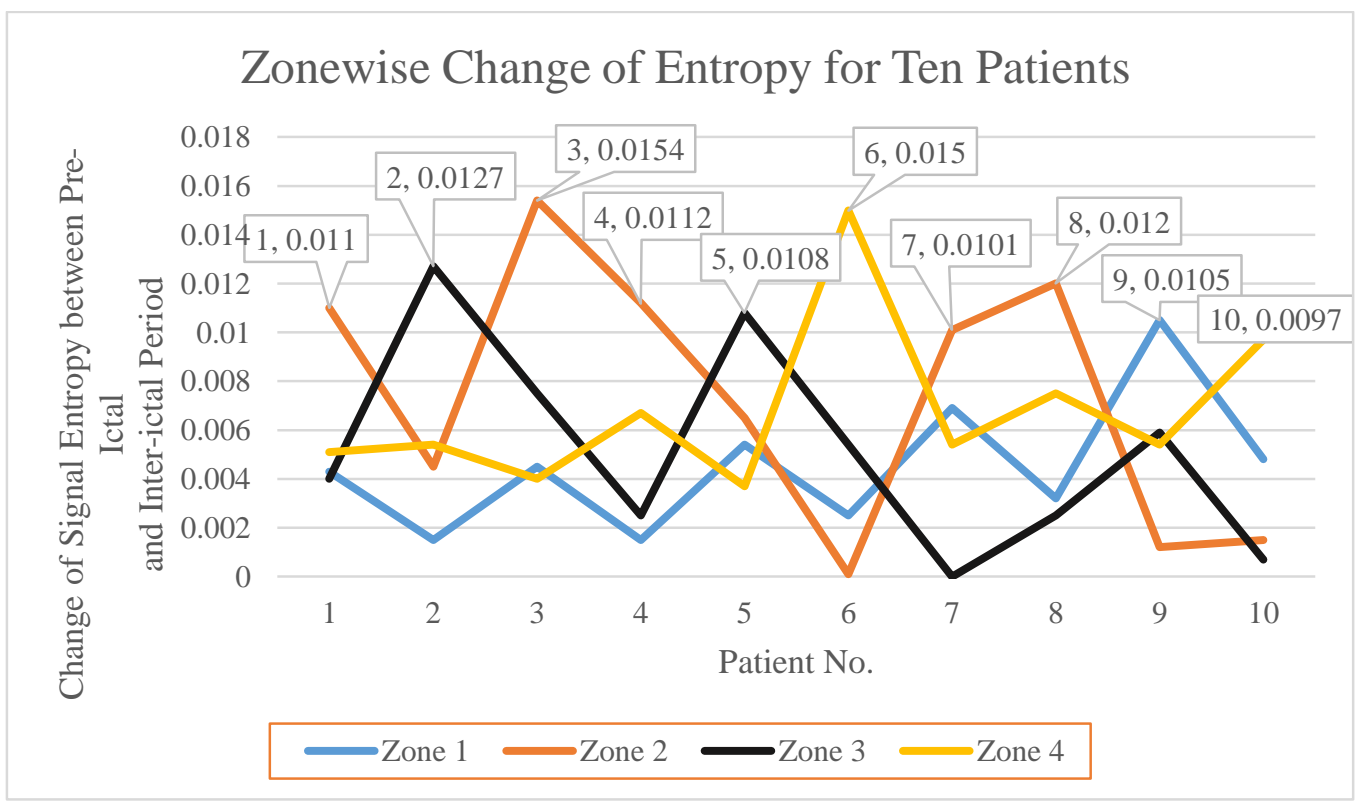

Figure 5. Change of Signal Entropy between Non-ictal and Pre-ictal period for ten patients. 
From Fig. 5, it can be observed that Patient 9 had significantly higher change of entropy in brain zone 1, i.e., epileptic activities were located under brain zone 1. Hence, it can be inferred that most of the epileptic activities were localized under brain zone 1 and the EEG electrodes under brain zone 1 can successfully demonstrate the onset of epileptic activity if the subject is not otherwise abruptly taken care of. Likewise, brain zone 2 localized the epileptic activity site for patient 1,3,7,8. Fig. 6 shows the localized brain region mapping of the comparative entropies as calculated in Table 1 for individual patients. The highlighted zones localized the regions of epileptic seizure activities for individual subjects.

\section{CONCLUSION AND FUTURE WORK}

The pre-ictal period has great importance in predicting epileptic seizure. The epileptic seizure can be predicted at onset with the abrupt change of signal entropy during pre ictal period and this research work gives an approach about this fact. A sudden change in entropy in the pre-ictal period was noticed clearly in the results and that was the focal point to predict the seizure early. Thus, this can be of great importance to stop the seizure from occurring by the use of medications and creating awareness in advance. Moreover, the region of the epileptic activities was also localized by dividing the total brain into four topographic regions and by calculating the entropy from this four zones separately. Thus, this findings have the potential to help the clinical neurologists to investigate seizure detection and treat the patient in a better way with less supervision and better accuracy.

This work was based on the data provided in the Physionet. The technique can be further implemented in future on real life cases to validate its applicability in clinical surroundings. 


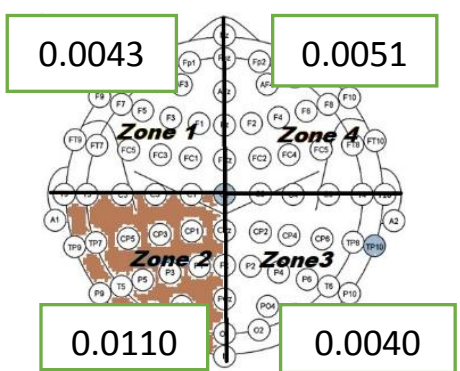

(a) Patient 1

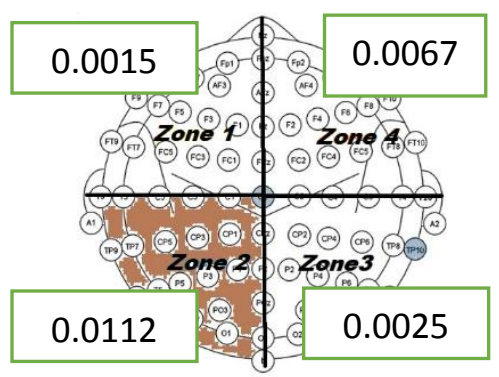

(d) Patient 4

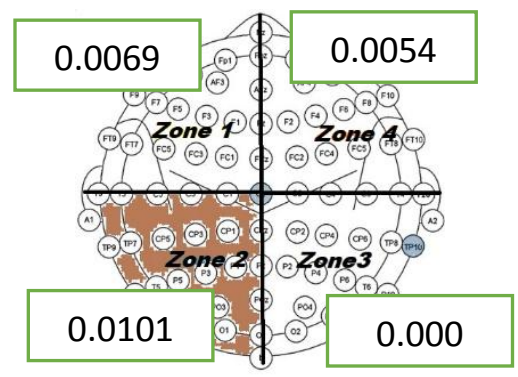

(g) Patient 7
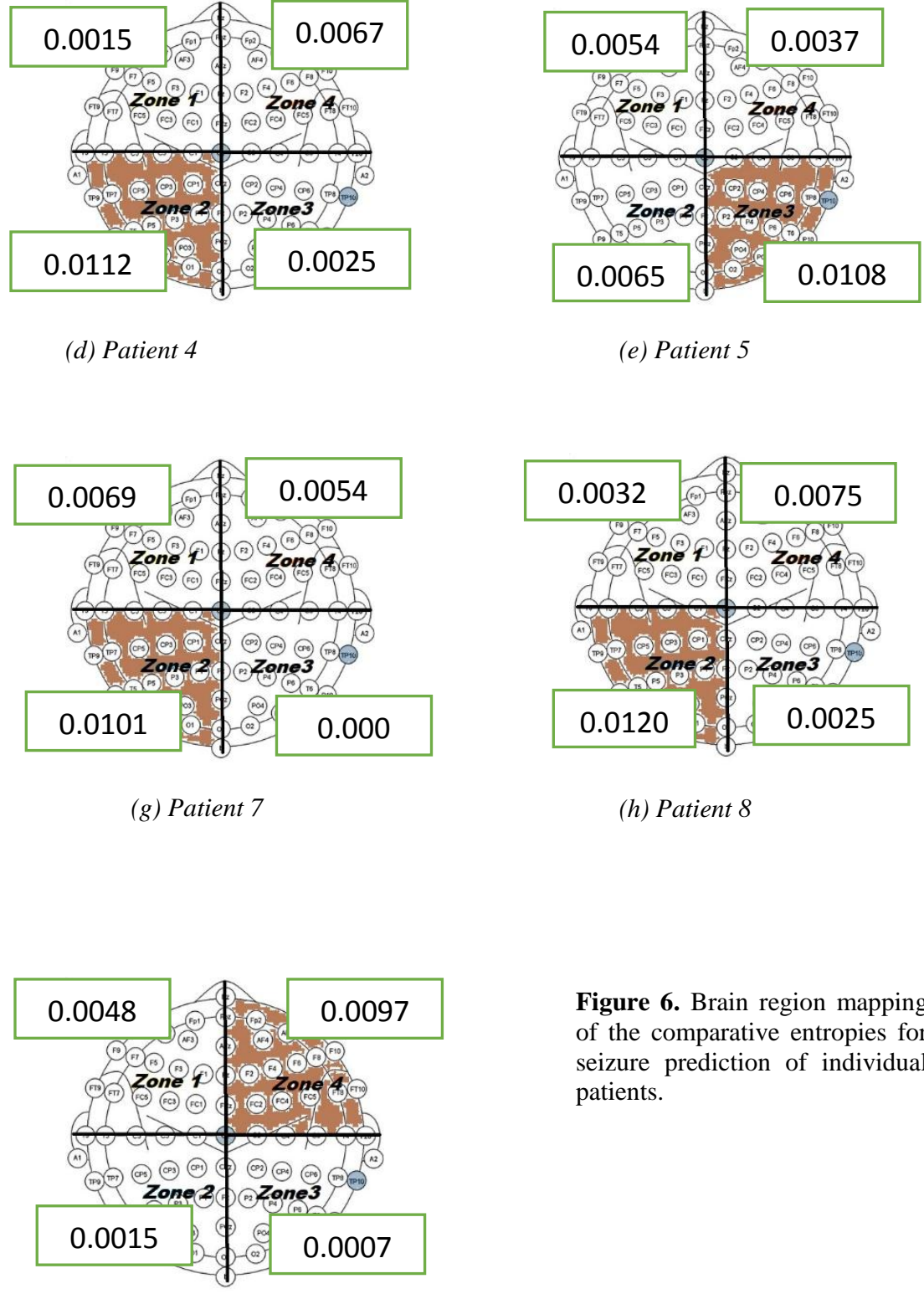

(e) Patient 5
Figure 6. Brain region mapping of the comparative entropies for seizure prediction of individual patients. (h) Patient 8

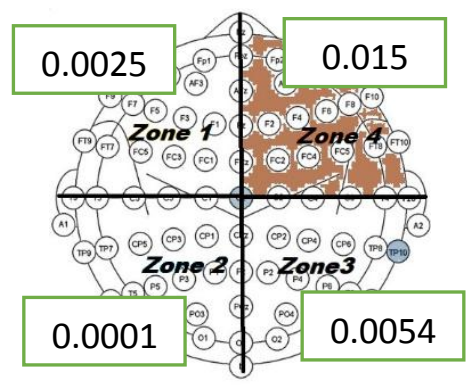

(f) Patient 6

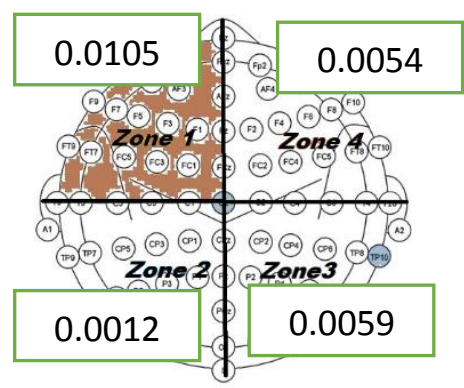

(i) Patient 9

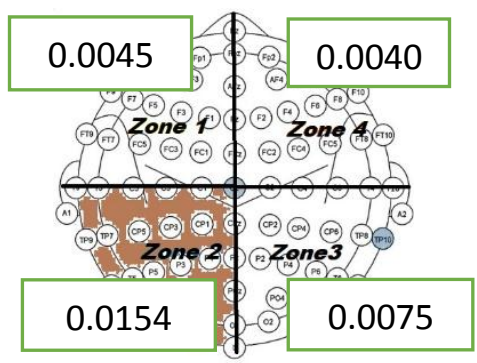

(c) Patient 3

(b) Patient 2

(j) Patient 10 


\section{REFERENCES}

Ahmad, R. F., Malik, A. S., Kamel, N. \& Reza, F., 2013. A proposed framework for real time epileptic seizure prediction using scalp EEG. IEEE International Conference on Control System, Computing and Engineering, pp. 284-289, Pennag, Malayasia.

Chaudhary, U. et al., December, 2012. Mapping preictal and ictal haemodynamic networks using videoelectroencephalography and functional imaging. Brain, 135(12), p. 3645-3663.

Dollahpour, A. \& Jalilifar, M., 2014. Seizure prediction methods: A review of the current predicting techniques. Journal of Biomedical and Neuroscience, Volume 7, pp. 153-162.

Goldberger, A. et al., 2000. PhysioBank, PhysioToolkit, and PhysioNet: Components of a New Research Resource for Complex Physiologic Signals. Circulation, 101(23), pp. 215-220.

Li, P. et al., 2018. Detection of epileptic seizure based on entropy analysis of short-term EEG. PLoS ONE, 13(3).

Ramgopal, S., Thome-Souza, S., Jackson, M. \& Loddenkemper, T., 2014. Seizure detection, seizure prediction and closed loop warning system in epilepsy. Epilepsy and Behavior, Volume 37, pp. 291-307.

Shannon, C. E., 1948. A mathematical theory of communication. Bell Systems Technical, Volume 27, p. 379-423.

Shufang, L., Weidong, Z., Qi, Y. \& L.Yinixia, 2013. Seizure prediction using Spike rate of Intracranial EEG. IEEE Transactions on Neural Systems and Rehabilitation Engineering, 21(6), pp. 880-886.

Teplan, M., 2002. Fundamentals of EEG Measurement. Measurement Science Review, Volume 2, pp. 1-11.

WHO, 2005. ATLAS: Epilepsy Care in the World. Geneva, Switzerland: World Health Organization. 